

\title{
DISCIAIRIF.R
}

\begin{abstract}
This documenl was prepared as an urctunt of work spomsored by an agency of the ['nited States Guternenent. Neither the I'nited Siates Government nor the I niversily of California nor ony of their employetes, mukes any warsanty. evpress os implied, or assumes any legal liability or responsihility fur the accuracy. completeness, or uscfulness of any infurmation, apparatus, product, or process disclosed, or represents that its use would not infringe privately ah ned rights. Kefference hescin to any sperific cummercial products, process, or service by trade name, Irademark. manufacturer. or itherwise, doxy not necessarily conslitult or imply its endoraement. recommendation. or farosing by the I'nited States Covernment or the l'niversity of California. The viens and opinions of authors expressed herein do not necessurily state or renecl those of the linited Stales Government ar the l niversily af ('alifnrnia, and shall nut he used for advertising ar product endorsiment purpenses.
\end{abstract}




\section{HIGH GRADIENT ACCELERATORS FOR LINEAR LIGHT SOURCES}

UCRL--99268-Rev. 1

William A. Barletta

DE89 001124

University of California, Lawrence Livermore National Laboratory, P.O. Box 808, Livermore, California* and Center for Advanced Accelerators, Department of Physics, University of California at Los Angeles

Ultra-high gradient radio frequency linacs powered by relativistic klystrons appear to be able to provide compact sources of radiation at XUV and soft $x$-ray wavelengths with a duration of 1 picosecond or less. This paper provides a tutorial review of the physics applicable to scaling the present experience of the accelerator community to the regime applicable to compact linear light sources.

\section{INTRODUCTION}

Linear light sources capable of producing picosecond bursts of XUV and soft $x$ radiation have gained ever increasing attention with the rapid advances both in free electron laser (FEL) physics and in the technology of ultra-high gradient linacs capable of producing high brightness electron beams at high energy. Compact accelerators for linear light sources are based upon conventional radio frequency structures. Desired average accelerating gradients $(\approx 200 \mathrm{MeV} / \mathrm{m}$ or more) are higher than those achievable in present-day accelerators (c.g., $17 \mathrm{MrV} / \mathrm{m}$ at SLAC). This goal is, however, being pursued actively at Lawrence Livermore National Laboratory (LLNL) in collaboration with the Stanford Linear Accelerator Center (SL.AC) and the Lawrence Berkeley Laboratory (LBL) toward the design of a TeV electronpositron collider. A great deal is known about the basic scaling laws govening the operation of rf accelerators. They are presented here as a tutorial guide to those considering the design of compart linear light sources (or flavor factories).

* Work performed under the auspices of the U.S. Departunent of Energy by Lawrence Livermore National Laboratory under contract W-7405-ENG-4S. 
A critical beam characteristic with respect to the performance of an $x$-ray FEL (XRFEL) is the emittance of the beam, because it strongly affects the gain of the laser. As discussed in Sec. 2 , design goals of $\varepsilon_{n} \approx 10 \mathrm{~mm}$-mrad and $N_{b} \approx 5 \times 10^{10}$ are consistent with the performance of existing electron sources. Constraints on beam chacteristics imposed by cavity fill times, focusing, beam loading, wakeficld control, and gas matching (in the case of plasma assisted wigglers) are presented in Secs. 3-6.

A major technological lurdle to be overcome in realizing high gradient rf accelerators is the gigawatt of power source. Section 7 discusses the inductior -iinacpowered relativistic klystron and the possibility of deploying branched maguetics in order to provide macro-pulses as long a $1 \mu$ s duration should the community of linear light source users require such a characteristic. Finally, Sec. 8 presents tho various elements of a cost estimation algorithm that can be used by the designer of a linear light source (or flavor factory) to access the financial resources needed to build a high gradient linac powered by relativistic klystrons.

\subsection{Application-Dervied Constraints}

Because of the diverse radiation output characteristics desired by the potential user communities, no specific facility or economic constraints have been fornulated for linear light sources. It is useful, nonetheless, for the accelerator designer to adopt some rougl guidelines as goals:

$$
\begin{aligned}
& \text { Length/energy }<5 \mathrm{~m} / \mathrm{GeV}, \\
& \text { Cost/encrgy/pulse duration }<10 \mathrm{M} \$ / \mathrm{GeV} / \mu \mathrm{s}, \\
& \text { Repetition rate }>200 \mathrm{~Hz} .
\end{aligned}
$$

Cast in this form the eost and size gorals for the light source designer are found to be roughly equivalent to these of the designer of the uext generation of linear colliders at TeV energies. Exploring design concepts consist ent with these goals lass 
been the object of active and steadily increasing efforts by the high encrgy physics research community throughout the world. Consequently, a substantial literature exists concerning the physics of accelerators relevant to compact linear light sources. Recent work by Wilson, ${ }^{1,2}$ and Palmer, ${ }^{3}$ and Amaldi ${ }^{4}$ form the basis of the analysis

of Secs. 2-6. A spread-sheet program including these analyses, those concerning cost, and those of FEL physics is now in active use at LLNL with a goal of system optimization.

\subsection{Energy Constraints}

In addition to setting sizc and cost coustraints, the physics of free electron laser operation set several constraints on the characteristics of the beam such as the beam energy, $E$ (or relativistic factor, $\gamma$ ), the normalized emittance, $\varepsilon_{n}$, and the energy spread, $\Delta E / E$ (or momentum spread, $\Delta p / p$ ). For an FEL with a planar wiggler the well-known design equations linking the beam characteristics with FEL performance are specified in terms of the wiggler field, $\boldsymbol{B}_{0}$, the wiggler wavelength, $\lambda_{w}$, and the radiation wavelength, $\lambda_{s}$. The wiggler characteristics can be combined in terms of a dimensionless vector potential

$$
K^{r}=\epsilon \lambda_{w}\left(B_{0}\right) / 2 \sqrt{2 \pi} m_{e} c^{2} \approx 0.66 B_{0}(T) \lambda_{u^{\prime}}(\mathrm{cm})
$$

The wiggler and radiation wavelengths are connected to the beam energy by the resonance condition

$$
\lambda_{s}=\left(\lambda_{w} / 2 \gamma^{2}\right)\left(1+H^{2}\right)
$$

\subsection{Mono-Chromaticity and Emittance Requirements}

FEL physies place tight bounds on an inuportant characteristic of the accelerator subsystem, namely, the allowable momentum sprearl, $\Delta p / p$, within the pulse or from bunch to bunch within a macro-pulse. The noment um spread bas several sources, the largest of which originates from the duration of cach pulse relative to 
that of the rf cycle. This spread, plus longitudinal wakeficld effects, will make it difficult to produce high energy beams with $\Delta p / p< \pm 0.1 \%$.

The performance of the FEL scales ${ }^{5}$ with a single electron beam parameter, $\rho$ :

$$
\rho=\left(\frac{\hbar \omega_{p}}{\delta \omega_{w}}\right)^{2 / 3},
$$

where $\omega_{p}\left(\sim \gamma^{-3} N_{b}^{1 / 2}\right)$ is the relativistic plasma frequency of the elcctron beam. After passing through a wiggler of length $L \sim \gamma / \rho$, the output from the FEL will saturate with the power in the radiation being $\rho$ times the electron beam power. The constraints on energy spread in the beam and the normalized beam emittanec are

$$
\frac{\Delta E}{E} \leq \frac{\rho}{4}
$$

and

$$
\varepsilon_{n} \leq \lambda_{s} \gamma
$$

The final constraint on the desing of the XRFEL comes from requiring that the gain length, $L_{g}$, of the laser be shorter than the Rayleigh range, $Z_{r}$. The gain length can be expressed in terms of the FEL parameter; namely,

$$
L_{g}=\frac{\lambda_{w}}{4 \pi \rho}
$$

the Rayleigh range is given by

$$
Z_{R}=\frac{\pi a^{2}}{\lambda_{s}}
$$

Hence,

$$
a^{2}>\frac{\lambda_{s} \lambda_{u}}{r \pi^{2} \rho}
$$

where $a$ is the size of the beam in the wiggler. If the wiggler employs natural focusing, the betatron wavelength in the wiggler and the beam size are related by

$$
\lambda_{\beta}=\frac{\lambda_{,} \gamma \sqrt{2}}{\kappa}
$$


The beam size and betatron wavelength are, in turn, related to the emittance by

$$
\epsilon_{n}=\frac{2 \pi \gamma a^{2}}{\gamma_{\beta}}
$$

If the XRFEL employs a plasma-assisted wiggler ${ }^{6}$ in which the beam assumes its self-focused radius in the presence of an ion channel (or if the beam is to be focused to an extremely small radius as in a flavor factory), a magnet subsystem must match the beam size in the accelerator $(\sim 100 \mu \mathrm{m})$ to the self-focused size in the plasma ( $\sim 1 \mu \mathrm{m})$. Relatively compact focal arrays with such capabilities have received considerable attention from the designers of linear colliders. Most notably, the Stanford Linear Collider (SLC) has such a final focus system in operation. For the SLC design, the size of the focal spot is related to the momentum spread by

$$
a_{\text {locus }} \propto\left(\frac{\Delta p}{p}\right)^{2}
$$

A focal system with quadrupoles with field strengths of less than $2 \mathrm{~T}$ and apertures of $1 \mathrm{~cm}$ will require that $\Delta p / p< \pm 0.5 \%$.

\section{CHARACTERISTICS OF EXISTING ELECTRON BEAM SOURCES}

This section reviews the present state of the art concerning bright, high current sources of electron beams. These sources, which include electron injectors, synchrotron radiation sources, and linear colliders, form the data base for scaling accelerator design to the regime applicable to linear light sources and flavor factories.

\subsection{High Brightness Sources}

As the beam emittance plays a critical role in determining electron density and thereby the gain of a free electron laser, the examination of the scaling basis for accelerators for lincar light. sources begins with a discussion of the present status of ligh brightness electron souress. Over the past decade the acelerator community has expended considerable effort in the design of ever lower cult tance sources of 
electron beams with large peak currents. This work has been motivated by three applications: high energy linear colliers, high average power FELs operating at visible or shorter wavelengt $\mathrm{l}_{\mathrm{h}} \mathrm{s}$, and effective sources of incoherent synchrotron radiation. During this period the beam brightness (current density divided by angular divergence) has been increased by at least three orders of magnitude, with prescntly achieved performances still a considerable distance from fundamental limits.

In early 1987 Brookhaven National Laboratory hosted a workshop ${ }^{7}$ concerning the production of low emittance beams for both collider and FEL applications. The most stressing goal, that for XRFELs as set for the participants by Pellegrini, was a factor of a few more demanding than that for linear colliders; namely,

$\begin{array}{ll}\text { Beam Energy, E } & 1 \mathrm{GeV}, \\ \text { Normalized horizontal emittance area, } \epsilon_{n x}=\gamma \epsilon_{z} & 1 \times 10^{-6} \pi \mathrm{m}-\mathrm{rad}, \\ \text { Normalized vertical emittance area, } \epsilon_{n y}=\gamma \epsilon_{y} & 1 \times 10^{-8} \pi \mathrm{m}-\mathrm{rad}, \\ \text { Longitudinal brilliance, } B_{L}=I_{\text {beam }} / \gamma & 200 \mathrm{~A} .\end{array}$

Working groups studying beam injectors, damping rings, and the problems of emittance preservation during the acceleration process concluded that the injectors would have to be improved bejond the present state of the art to meet the stated goa!s for an FEL. They found no mechanism incompatible with this goal and expected that such a beam could be accelerated from a few tens of MeV to a few GeV without degradation.

The best perfornance in building high current, low emittance guns using photocathodes has been achieved by Fraser, Sheffielel et al ${ }^{8,9}$ at Los Alamos. They have produced a beam at $1.1 \mathrm{MeV}$ with a peuk current of $130 \mathrm{~A}$ and a normalized rms emittance of $5 \times 10^{-6} \pi \mathrm{m}$-rad. This value is approximately twenty times smaller than that of the SLAC gun,${ }^{10}$ which produces a beam of $\approx 1 \mathrm{kA}$ at an enittance of $2 \times 10^{-4} \pi \mathrm{m}$-rad. At SLAC this benm is accelerated to $\approx 1 \mathrm{GrV}$ for insertion 
into a damping ring. Upon exiting the damping ring the beam emittance has been reduced to $1 \times 10^{-6} \pi \mathrm{m}$-rad.

Low emittance beams have also been produced with thermionic cathodes in If guns, albeit at somewhat lower peak currents. For example, the Mark III used for FEL experiments at Stanford's High Energy Physics Laboratory delivers a peak current of $30 \mathrm{~A}$ at $44 \mathrm{MeV}$ with normalized horizontal and vertical emittances of $\varepsilon_{n x}=3.6 \times 10^{-6} \pi \mathrm{m}$-rad and $\varepsilon_{n y}=1.8 \times 10^{-6} \pi \mathrm{m}-\mathrm{rad}$, respectively.

\subsection{Existing and Planned Synchrotron Beams}

Existing and planned synchrotron sources ${ }^{11}$ in the USA are (in alphabetical order) Argonne $6 \mathrm{GeV}$ Ring, Berkeley 1-2 GeV Ring, Cornell High Energy Synchrotron Source (CHESS), National Synchrotron Light Source (NSLS) (Brookhaven), Stanford Synchrotron Radiation Laboratory (SSRL), Stanford Phoion Research Laboratory (SPRL), Synclirotron Radiation Center (SRC) (Stoughton), and Synclirotron Ultraviolet Radiation Facility (SURF) (Gaithersburg). Their properties are detailed in Table 1.; note that the emittances given are unnormalized, i.e., $\varepsilon=\varepsilon_{n} / \gamma$.

\subsection{The Stanford Linear Collider (SLC)}

The most relevant data base for scaling the design of if linac technology to the parameter regime suitable for linear light sources (and for linear collider flavor factories) is the $50 \mathrm{GeV}$ SLC at the Stanford Linear Accelerator Center (SLAC). The SLC linac consists of a $3-k i n$-long assembly of sections of S-band $(2.87 \mathrm{GHz})$ disk-loaded waveguide (traveling wave). Each linac section, consisting of nearly 100 cavitics with a length one-third the if wavelength, is fed by a ligh pridi power klystron. Although other types of $r$ structures (c.g., standing wave) may also br suitable, the disk-loaded structure is menable to analytical calculation and has received considerable study. Consecguently, scaling the SLC design is a convenient way to scope the characteristics of the accelerator. The following analysis requires 
Table 1. Existing and Planned Storage Rings

\begin{tabular}{|c|c|c|c|c|}
\hline Status* & Name & $\begin{array}{c}\text { Energy } \\
(\mathrm{GeV})\end{array}$ & $\begin{array}{l}E_{\text {horis }} \\
(\pi \times\end{array}$ & $\begin{array}{l}\varepsilon_{\text {vert }} \\
\text { rad) }\end{array}$ \\
\hline ò & ALADDIN (Stoughton) & 0.75 & 63 & 110 \\
\hline o & TANTULUS (Stoughton) & 0.24 & 230 & 4000 \\
\hline $\mathbf{p}$ & ALS (LBL) & 1.3 & 6.8 & 4.0 \\
\hline $\mathrm{p}$ & ANL (Argonne) & 7.0 & 7.0 & 0.2 \\
\hline$\circ$ & CESR (Cornell) & 5.5 & 200 & 6.6 \\
\hline o & NSLS I(BNL) & 0.75 & 130 & 230 \\
\hline$\circ$ & NSLS II(BNL) & 2.5 & 80 & 13 \\
\hline \multirow[t]{2}{*}{$\circ$} & PEP (SLAC) & 15 & 150 & 0.67 \\
\hline & $"$ & 8.0 & 10 & 0.16 \\
\hline$\circ$ & SPEAR (SLAC) & 3.0 & 450 & 50 \\
\hline o & SURF II(Gaithersburg) & 0.28 & 270 & 3400 \\
\hline c & SPRL (SLAC) & 1.0 & 10 & 10 \\
\hline
\end{tabular}

- $o=$ operational, $c=$ in construction, $p=$ planned

the definition of several quantities listed in Tuble 2. along with their respective values for the SLC. Units are mks unless noted.

\section{SCALING BASIS FOR ACCELERATORS FOR LINEAR LIGHT SOURCES}

For use in a linear light source, a compact accelerator should both be highly efficient in transferring of encrgy from the source to the high energy beam and highly conservative in use of plyysical space. As shown in the next section, these constraints push one toward high gradients, high frequencies, and short fill times. 
Table 2. SLC values of scaling variables

\begin{tabular}{lll}
\hline & $E$ & $50 \mathrm{GeV}$ \\
Beam energy & $f$ & $2.87 \mathrm{GHz}$ \\
RF frequency & $\lambda$ & $10^{5} \mathrm{~mm}$ \\
RF wavelength & $a$ & $11 \mathrm{~mm}$ \\
Iris aperture (radius) & $Q$ & $1.39 \cdot 10^{4}$ \\
Cavity Q & $v_{g}$ & $0.011 \mathrm{c}$ \\
RF Group velocity & $t_{f}$ & $921 \mathrm{~ns}$ \\
Fill time & $L_{f}$ & $2.97 \mathrm{~m}$ \\
Length per feed & $T_{o}$ & $1.4 \mu \mathrm{s}$ \\
Attenuation time & $t$ & 0.6 \\
Fill length / attenuation & $E_{a}$ & $17 \mathrm{MeV} / \mathrm{m}$ \\
A verage gradient & $E_{\max }$ & $41.3 \mathrm{MeV} / \mathrm{m}$ \\
Peak field in cavity & $h_{b}$ & $3.12 \%$ \\
Loading & $P_{r f}$ & $0.8 \mathrm{MW} / \mathrm{m}$ \\
Peak RF power & $N_{b}$ & $\leq 5 \times 10^{10}$ \\
Particles per bunch & $s_{z}$ & $1 \mathrm{~mm}\left(3.43^{\circ}\right)$ \\
Bunch length (Gaussian $\sigma)$ & $\sigma_{p}$ & $0.39 \%$ \\
Momenturn spread $(\Delta p / p)$ & $\sigma_{x, y}$ & $77 \mu \mathrm{m}$ \\
Beam size in linac & & \\
\hline
\end{tabular}

\subsection{High Voltage Breakdown}

The physical phenomenon which forms the basis for sealing of linacs from the $S L C$ value of $17 \mathrm{MeV} / \mathrm{m}$ to the required $>200 \mathrm{MeV} / \mathrm{m}$ is the observation ${ }^{12}$ that the peak electric field that can be sustained without breakdown rises with increasing of frequency and with shortening duration of the of power (sec Fig. 1).

Not all of the potential for increased fields cnn be realized in practical high 


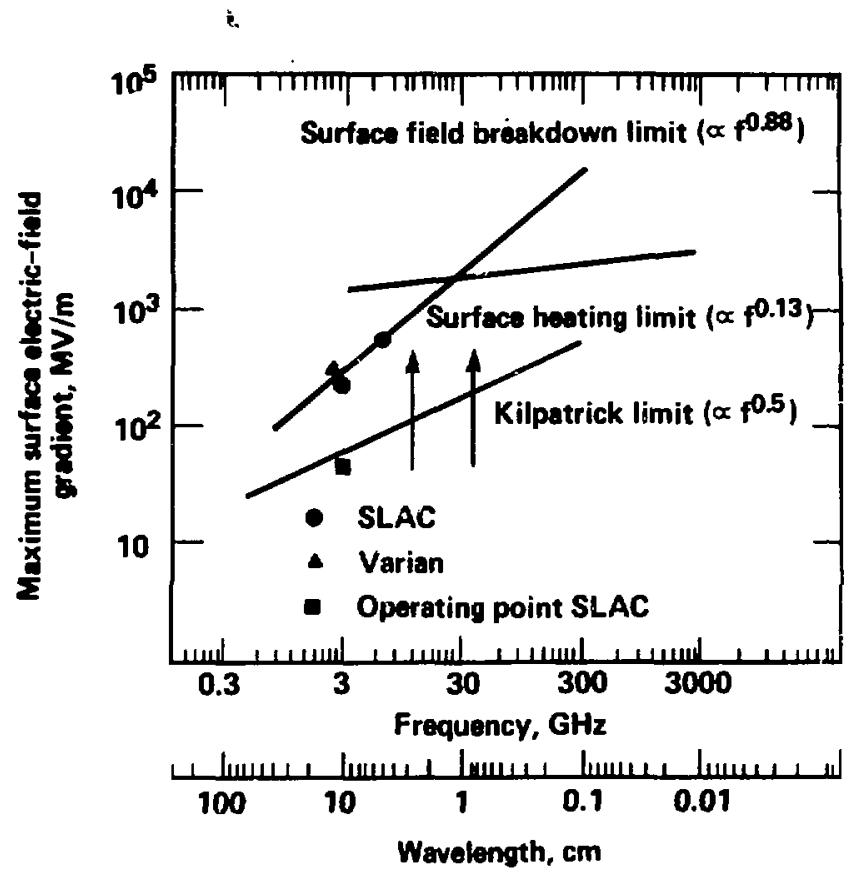

Figure 1. The peak electric field that can be sustained in if structures without breakdown. Generally, only approximately half this value can actually be used.

energy accelerators. At field values somewhat below breakdown values, small currents will flow near the cavity walls. These currents give rise to copious $\mathrm{x}$ radiation and, more significantly, to magnetic fields that exert random transverse forces on the beam, thereby exciting wakefield instabilities. To avoid this difficulty the peak field should be reduced by a safety factor, $F_{s}$, with $0.5 \leq F_{s} \leq 0.75$. The interdependence model uses a value $F_{s}=0.66$. Commonly, in the discussion of linear colliders, the scaling of $E_{p k}$ with frequency is given as $E_{p k} \propto f^{7 / 8}$. This scaling takes advantage of the increase of sustainable peak field with decreasing if pulse 
duration:

$$
E_{p k}=120 \mathrm{MV} / \mathrm{m}\left(\frac{F_{a}}{0.66}\right) \cdot\left(\frac{f}{2.87 \mathrm{GHz}}\right)^{7 / 8}
$$

Of course, this value can only be achieved if sufficient power is supplied to the structure; it is not in the case of SLC. Should the application require an output period of $\sim 1 \mu s$ indpendent of frequency, the exponent in Eq. 6 should be reduced to $1 / 2$.

\subsection{Cavity and if Characteristics}

The scaling characteristics of $2 \pi / 3$ disk-loaded structures as described in Refs. 1-3, 13, and 14 are based on computer calculations with Farkas's code TWAP. The disk-loaded structure has a phase velocity equal to $c$, the speed of light, and a group velocity that is a function of the rf wavelength and the aperture of the iris. Palmer's fits $^{3}$ to the TWAP calculations ${ }^{13}$ as shown in Fig. 2(b) give

$$
\beta_{g}=\frac{v_{g}}{c} \approx \exp \left[3.1-2.4\left(\frac{\lambda}{a}\right)^{1 / 2}-0.9\left(\frac{a}{\lambda}\right)\right]
$$

Witl the group velocity specified the remainder of the cavity characteristics can be calculated. Initially assume that no of encrgy is lost as the wave travels through the structure. In that case the energy per unit length, $u_{a}(\mathrm{~J} / \mathrm{m})$, required 

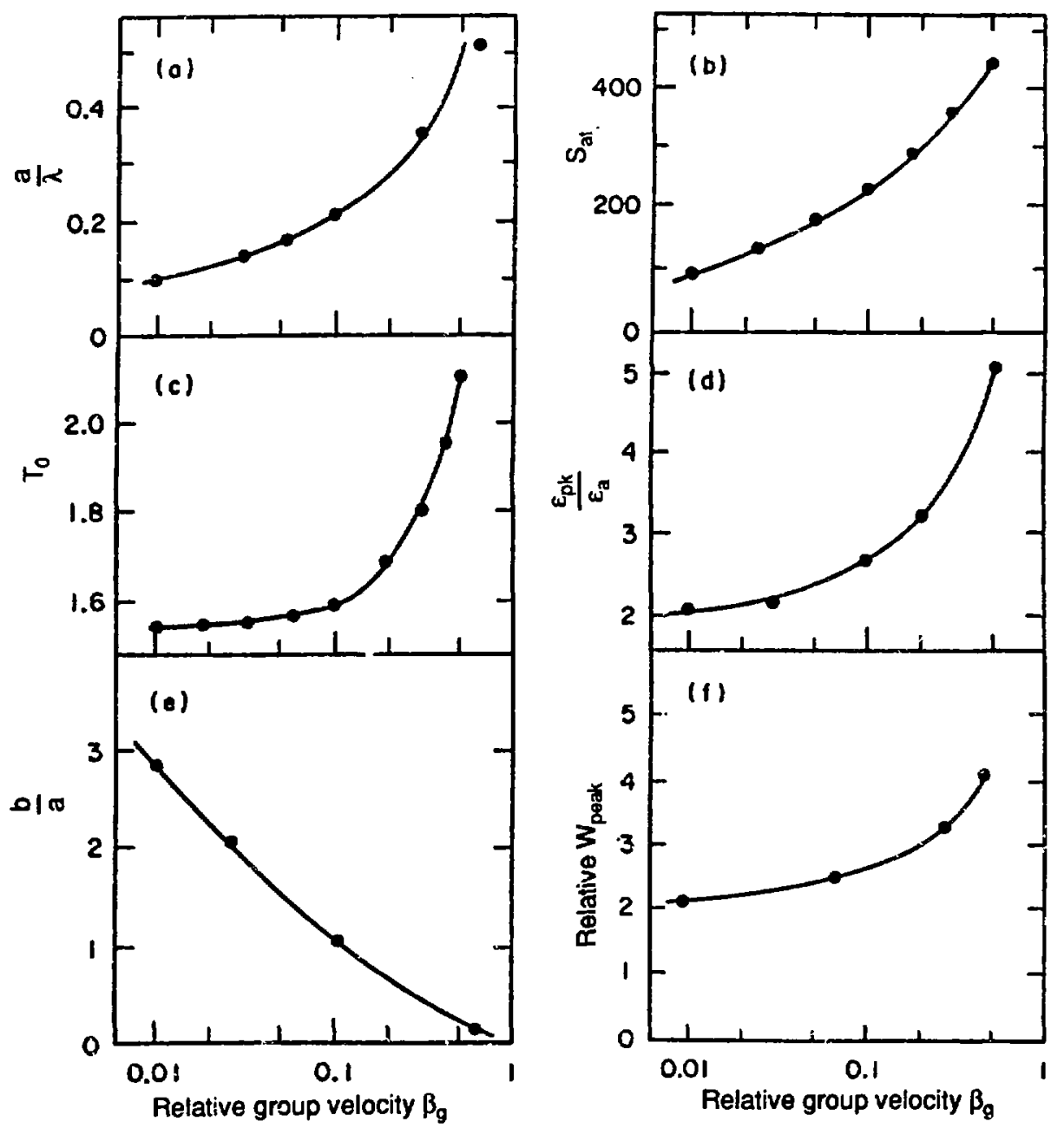

Figure 2. Palmer's ${ }^{3}$ fit 3 to Farkas's TWAP ${ }^{13}$ calculations of SLAC-like accelerating structures. (a) iris radius $a$ divided by wavelength; (b) normalized corrected elastance $S_{a t} ;$ (c) attenuation time constant $T_{0}$ in $\mu \mathrm{sec}$; (d) ratio of peak and average accelerating fields; (e) ratio of outer cavity and iris radii; (f) the relative peak if power. 
to produce a given acceleration of the beam is related to the average accelerating gradient by a monotonically increasing function of the group velocity, namely, the corrected elastance, $s_{t}$ :

$$
s_{t}=\frac{E_{a}^{2}}{w_{a}}=5.7 \cdot 10^{14} \mathrm{~V} / \mathrm{mC}\left(\frac{10 \mathrm{~mm}}{a}\right)^{2}\left(\frac{v_{g}}{c}\right)^{0.4} .
$$

Frequently accelerator designers prefer to quote a "normalized" elastance that is independent of the iris aperture, i.e.,

$$
s_{a t}=s_{1} a^{2}
$$

Because both the beam and the of move through the structure with a finite velocity, the length of the accelerating pulse can be less than that of the section. Therefore, the amount of energy, $w_{\rho}$, needed to fill the structure is greater than $w_{a}$ by an amount

$$
w_{f}=\frac{w_{a}}{\left(1-\beta_{g}\right)} \text {. }
$$

Similarly, the uncorrected elastance is related to $s_{t}$ by

$$
s=\left(1-\beta_{g}\right) s_{i} .
$$

In actual structures the of wave is attenuated as it travels down the section. The characteristic attenration time,

$$
T_{o}=1.45 \mu \mathrm{s}\left(1+1.20 \beta_{\mathrm{g}}^{1.5}\right)\left(\frac{\lambda}{105 \mathrm{~mm}}\right)^{1.5},
$$

is related to the $Q$ of the cavities by

$$
Q=\pi f_{r f} T_{o}
$$

The fill time of the section is

$$
t_{f}=\frac{L_{f}}{v_{g}}
$$


to produce a given acceleration of the beam is related to the average accelerating gradient by a monotonically increasing function of the group velocity, namely, the corrected elastance, $s_{t}$ :

$$
s_{t}=\frac{E_{a}^{2}}{w_{a}}=5.7 \cdot 10^{14} \mathrm{~V} / \mathrm{mC}\left(\frac{10 \mathrm{~mm}}{a}\right)^{2}\left(\frac{v_{g}}{c}\right)^{0.4}
$$

Frequently accelerator designers prefer to quote a "normalized" elastance that is independent of the iris aperture, i.e.,

$$
s_{a, t}=s_{t} a^{2}
$$

Because both the beam and the of move through the structure with a finite velocity, the length of the accelerating pulse can be less than that of the section. Therefore, the amount of energy, $w_{f}$, needed to sll the structure is greater than $w_{a}$ by an amount

$$
w_{f}=\frac{w_{a}}{\left(1-\beta_{g}\right)} .
$$

Similarly, the uncorrected elastance is related to $s_{\ell}$ by

$$
s=\left(1-\beta_{g}\right) s_{\ell}
$$

In actual structures the of wave is attenuated as it travels down the section. The characteristic at tenuation tine,

$$
T_{o}=1.45 \mu \mathrm{s}\left(1+1.29 \beta_{\mathrm{g}}^{1.5}\right)\left(\frac{\lambda}{105 \mathrm{~mm}}\right)^{1.5},
$$

is related to the $Q$ of the cavities by

$$
Q=\pi f_{r f} T_{0}
$$

The fill time of the section is

$$
\begin{gathered}
t_{f}=\frac{L_{f}}{v_{g}} . \\
-14-
\end{gathered}
$$


In terms of the ratio of the fill time to attenuation time,

$$
t=\frac{t_{f}}{T_{0}}
$$

the rf energy needed for the same acceleration in a constant impedance structure is

$$
w_{r f}=w_{a}\left[\frac{\tau^{2}}{\left(1-e^{-r}\right)^{2}}\right]=\frac{w_{a}}{h},
$$

w. $\quad a$ is defined as the structure efficiency. Note that as the length of the section is decreased the fill time goes to zero, but $w_{r f} \rightarrow w_{a}$. Therefore, the required power increases without bound:

$$
P_{r f}=u_{r f} / t_{f}
$$

Combining Eq. (7) with eq. (15), one can express the rf power per unit length as

$$
P_{\tau f}=\frac{E_{\mathrm{u}}^{2} a^{2}}{h T_{\mathrm{o}} \tau s_{a t}}
$$

The maximum gradient is determined from the peak permissible value of the electric ficld:

$$
E_{a}=\frac{E_{p k}}{2+6 \beta_{g}}
$$

where $E_{p k}$ is given hy Eq. (6). Hence,

$$
E_{a}=1.2 \cdot 10^{8} \mathrm{~V} / \mathrm{ln}\left(2+6 \beta_{g}\right)^{-1}\left(\frac{F,}{0.60}\right)\left(\frac{f}{2.87 \mathrm{GHz}}\right)^{1 / 2} .
$$

In designing a linear light source of minimum length for a given beam energy, one can choose only the frequency, iris aperture, and the attemation parameter; all the other cavity clanracteristics are deternined. Completing the cavity parameter descriptions is

$$
\frac{b_{c}}{a} \approx 1.04-0.20 \ln \beta_{g}+0.068 \ln ^{2} \beta_{g},
$$

where $b_{c}$ is the inner radius of the accelerating cavity. 


\section{FOCUSING IN THE LINAC}

The design of the focusing system for a high energy linac has two impacts. The first and least important of these is to increase the overall length of the accelerator. In a transport design scaled from the SLC FODO lattice this consideration can be quantified by a single number, $F_{q}$, the fraction of the linac occupied by the quadrupoles (typically $<10 \%$ ). The second implication is much more important. The strong focusing needed to control wake field instabilities can lead to severe tolerance constraints on the linac design. The beam transport in the linac is described by the beta function of the focusing lattice. The average strength of the focusing in a FODO structure consisting of quadrupoles with ficld strength $B_{q}$ and aperture $a_{q}$ is

$$
\left\langle\beta_{v}\right\rangle=\left(\frac{\sin \mu}{\mu^{2}} \frac{E}{e c} \frac{2 \varepsilon_{q}}{B_{q} F_{q}}\right)^{1 / 2},
$$

where $2 \mu$ is the phase advance per cell. Following the design of the SLC linac, one chooses

$$
\begin{aligned}
\mu & =45^{\circ}, \\
B_{q} & =1.5 \mathrm{~T} \\
a_{q} & =1.2 \mathrm{a} .
\end{aligned}
$$

With these choices one can rewrite Eq. (20) as

$$
<\beta_{y}>=2.49 \mathrm{nl}\left(\frac{E}{10 \mathrm{GeV}} \frac{a}{10 \mathrm{~mm}} \frac{1.5 \mathrm{~T}}{B_{q}} \frac{0.1}{F_{q}}\right)^{1 / 2} .
$$

Of course, the local viahe of the focusing strength will vary throughout the accelerator as the bcim gains energy. Using $\zeta$ to denote distance along the accelerator, one can compute the number of quadrupoles in the necelerator:

$$
N_{q}=\int_{0}^{L} \frac{d \zeta}{\beta(\zeta)^{\prime L}}
$$


where $L$ is the length of the accelerator. The beam size produced by this lattice is

$$
\sigma_{y}(\zeta)=\left(\frac{\beta_{y}(\zeta) \varepsilon_{n}}{\gamma(\zeta)}\right)^{1 / 2}
$$

Palmer ${ }^{3}$ shows that the alignment tolerances along the linac can be kept constant, if the strength of the quadrupoles is varied weakly $\left(\approx \gamma^{1 / 3}\right)$ along the linac; whence,

$$
\beta(\zeta) \propto \gamma^{1 / 3} \propto \zeta^{1 / 3}
$$

Then, Eq. (23) gives

$$
N_{q}=1.5\left(\frac{L}{\beta(L)_{\mu}}\right)
$$

Calculations ${ }^{13}$ of the ratio of beam displacement to beam radius at the end of the linac indicate that in order to preserve the beam emittance, the quadrupoles must be aligned with an rms accuracy of

$$
<d y>=\frac{\sigma_{y}}{\mu \sigma_{p}}\left(\frac{2}{N_{q}}\right)^{1 / 2},
$$

where $\sigma_{p}$ is the momentum spread, $(\Delta p / p)$. Probably the largest source of momentum spread in the main body of multi-GeV linacs will be a head-to-tail energy variation which is deliberately introduced and maintained throughout the accelerator in order to damp trasiverse wakefield effects. This technique is often erroneously called Landau damping, which refers to an instantaneous spread in betatron frequencies. The head-to-tail spread with the $4 k_{\beta}$ increasing at the tail is an idea of Balakin, Novokhatskii, and Smirnov and should be called BNS damping. The linear component of the head-to-tail spread can be removed in a small accelernting section at the end of the acceleritor that operates at a phase advance of $90^{\circ}$. This section has a length

$$
\begin{aligned}
l_{\text {cors }} & =\frac{\sigma_{p} p}{2 \pi E_{a} \sigma_{x}} \\
& =0.81 \mathrm{~m}\left(\frac{E}{10 \mathrm{GcV}}\right)\left(\frac{\lambda}{100 \mathrm{~mm}}\right)\left(\frac{E_{a}}{200 \mathrm{MeV} / \mathrm{m}}\right)^{-1}\left(\frac{\sigma_{z}}{10 \mathrm{~mm}}\right)^{-1},
\end{aligned}
$$


where $\sigma_{x}$ is the rms bunch length.

Wilson ${ }^{2}$ has showed that the tolerance on alignment of the focusing system in large linacs is generally more severe than the tolerance on alignment of the accelerator sections. A prudent approach, however, may be to adopt Eq. (27) as establishing the tolerance requirements for all beamline components. Similarly the position of the beam in the linac must be controlled to this level. For linacs of applicability to linear light sources and flavor factories, the alignment tolerances should not be worse than $\approx 100 \mu \mathrm{m}$. The difficulty may be compounded by the fact that vibrational tolerances in such linacs tend to be an order of magnitude tighter than the static alignment tolerances. Tolerance requirements may be relaxed if alternatives to BNS damping such as if focusing with elliptical irises prove practical; however, such means of controlling wake fields in high gradient linacs must still be considered to be untested at this time.

\section{WAKEFIELDS AND BEAM LOADING}

In passing through the accelerating structure each beam pulse will both remove energy from the fundamental accelcrating mode of the cavity and excite ligher order modes in the cavity. Via the excitation of these higher order modes (walicfields), the head of a beam pulse can affect the energy of (longitudinal wakes) and can deflect (transverse modes) the tail of the pulse. The results will be an energy spread in the beam and a head-to-tail growtl of emittance as the beam travels through the accelcrator.

\subsection{Transverse Wakefields}

The dipole (deflecting) modes lave an $E_{z}$ component that varies lincarly wit la distance from the cavity axis and with tlue cosine of the axinuthal angle, $f$. The nth traveling wave modes generated by a charge $q$ traveling parallel to the cavity 
axis at a radius $r_{\mathrm{g}}$ have an electric field at a distance $\zeta=c t$ behind $q$ of

$$
E_{z \pi}=-2 k_{n} q\left(\frac{r}{a}\right)^{m-1}\left(\frac{r_{q}}{a}\right)^{m} \cos m \phi \cos \omega_{n} \tau,
$$

where $k_{n}$ is Wilson's' loss parameter which relates the field intensity to the stored energy for the $n$th mode ( $E_{n}^{2} / W_{n}$ or one-fourtl the elastance for the mode). The interaction of the beam with these modes can be characterized by a transverse shunt impedarice $r_{\perp}$. For typical disk-loaded structures

$$
\frac{r_{\perp}}{Q} \approx \frac{100}{\lambda} \mathrm{ohms}
$$

gives a rough estimate of the transverse shunt impedance. According to Wilson, ${ }^{1}$ the lowest order (beam break-up) mode has a frequency $\approx 40 \%$ higher than the fundamental accelerating mode. Therefore, one expects the $Q$ of the dipole mode to be only slightly lower than that of the accelerating mode. Consequently, without special precautions the transverse wakefield of a pulse will persist for many tens of nanoseconds. Via these long-range wakes, both the transverse motion and the emittance can increase from one micro-pulse to another.

A theorem by Panofsky and Wenzel shows that the total deflecting kick on the particle at $\zeta$ can be expressed solely in terms of the $E_{z n}$ (or the corresponding potentials). The difficulty however, in computing the transverse deflection of the particle is that one must include as many modes as possible. The values of $\omega_{n}$ and $k_{n}$ in Eq. (29) are obtained by solving the boundary value problem for a charge-free cavity or structure with a computer code such as SUPERFISH. ${ }^{16}$ Figure 3 shows the results of such calculations of the dipole wake per unit accelcrating cell for the SLAC disk-loaded structure for three different time durations. In particular, Fig. 3(a) indicates the size of the contribution of the higher modes to the tatal dipole wake.

For the purposes of estimating the characteristics of high gradient linacs for linear colliders, Palmer ${ }^{3}$ lass fitted such computer calculations of the initial linear 

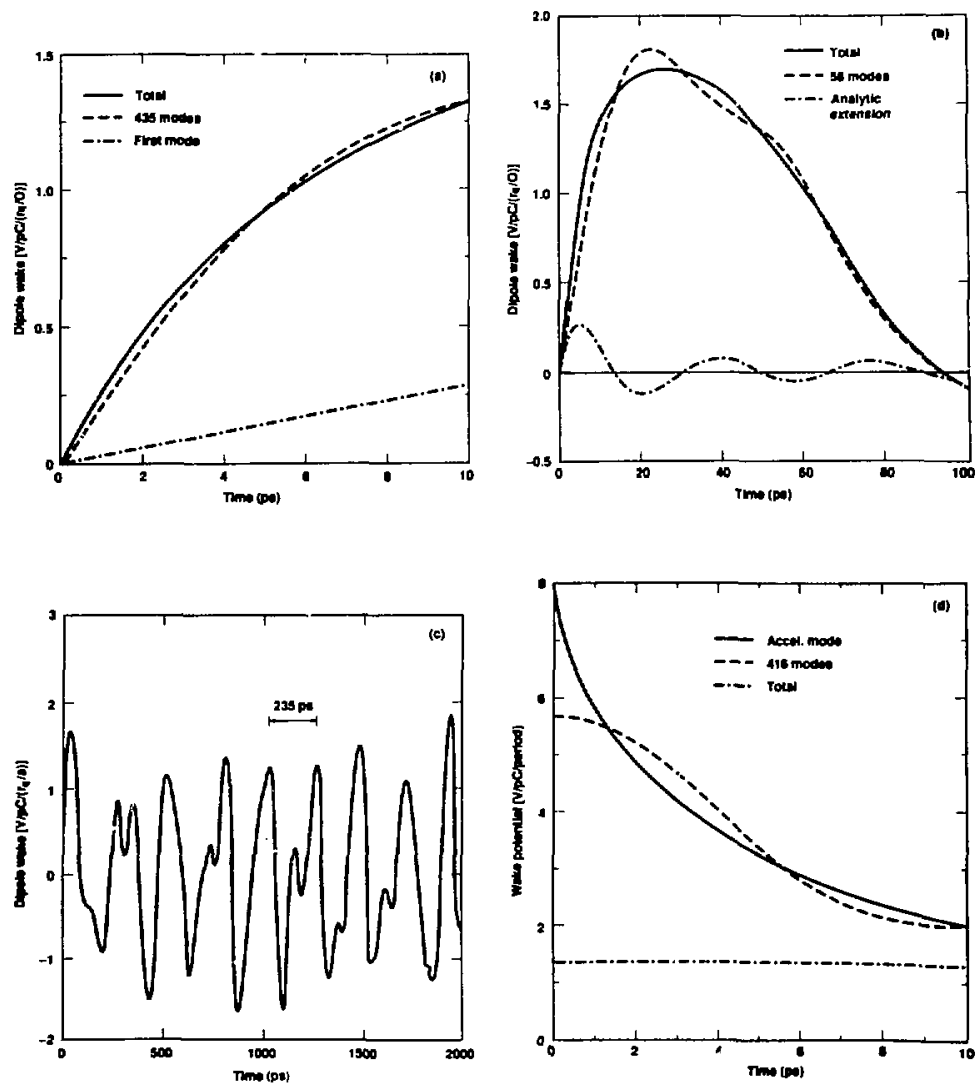

Figure 3. (a)-(c): Dipole walie per cell for the SLAC disk-loaded structures over vari us time intervals; (d) longitudinal wake per cell with a cell length of $3.5 \mathrm{~cm}$ and benm aperture radius of $1.163 \mathrm{~cm}$.

rise, ${ }_{1} W_{t}(\zeta)$, and the maximum of the wakefield, ${ }_{2} W_{t}$, to obtain the scaling laws (in mks units):

$$
\begin{gathered}
{ }_{1} W_{l}(\zeta)=6.64 \cdot 10^{10} \mathrm{~V} /\left(\mathrm{C}-\mathrm{m}^{2}\right) \zeta a^{-7 / 2} \lambda^{-1 / 2} \\
-20-
\end{gathered}
$$


for $\zeta \ll a$ and

$$
{ }_{2} W_{t}(\zeta)=3.28 \cdot 10^{10} \mathrm{~V} /\left(\mathrm{C}-\mathrm{m}^{2}\right) a^{-2.2} \lambda^{-0.8},
$$

which occurs at $\zeta \approx a$. For the SLAC geometry a reasonable fit to $W_{\ell}$ at intermediate values of $\zeta<a$ is obtained from

$$
W_{t}(\zeta)=\left[{ }_{1} W_{t}^{-2}(\zeta)+{ }_{2} W_{t}^{-2}(\zeta)\right]^{-1 / 2}
$$

As one can see from Fig. 3(c), the dipole fieids persist long after each beam pulse. Using an asymptotic approximation valid for long pulse trains, Yokoya ${ }^{17}$ has computed the value of the the dipole wakefield for multi-bunch macro-pulses in long linacs. He finds that the multi-bunch wake field has the same form as the single bunch wake with the growth determined by the average current during the macro-pulse. For the case of no spread in betatron frequency and strong focusing $\left(k_{\beta}=1 \mathrm{~m}^{-1}[E / 2 \mathrm{GeV}]^{-2 / 3}\right)$, a fit to Yokoya's calculations of the wake of the $j$ th pulse on the kth of cycle yields

$$
W(j)=0.30_{2} W_{1} j^{-0.8} \exp \left[\frac{-k}{Q^{*}}+1.38 j^{1 / 2}\right],
$$

where ${ }_{2} W_{t}$ is given by $\mathrm{E}_{\mathrm{q}}$. (32) and $Q^{*}$ is the effective quality factor of the dipole modes. This expression is overly pessimistic for short $(\approx 1 \mathrm{GeV})$ linacs designed to accelerate $\sim 10$ pulses in a macro-bunch.

As argued above, $Q^{*}$ is approximately $70 \%$ of the value for the fundamental $\left(\approx 10^{4}\right)$; hence, the decay term is of little help in preventing disastrous growth of the dipole mode. However, the frequency difference between the deflecting modes and the fundamental accelerating mode can, in prinriple, be used to out-couple the energy of these modes. Such a procedure is used in low-frequenry cw linars and has been cold-tested for disk-loaded stnuctures by Paluner and his collaborators at Brookhaven and SLAC. They lane becu able to design structures with a $Q$ of 20 for 
the dipole modes. In assessing system requirements for a XRFEL accelerator, one can characterize the requisite level of energy out-coupling by a dequeing parameter, $D$, where

$$
Q^{*}=0.7 \frac{Q}{D}
$$

This requirement is easily satisfied by ine slotted structures cold-tested at BNL.

5.1.1. Damping transverse motion. The transverse wakes from a single pulse can be effectively controlled by BNS damping the transverse motion through the introduction of a spread in betatron frequency increasing from head-to-tail within the beam. This spread can be obtained by appropriately centering the pulse in phase with respect to the if so as to maintain an energy spread between the front and back of the bunch. If the bunch is approximated as two point charges separated by $2 \sigma_{z}$, the energy spread required is

$$
\Delta E \approx 2 E \sigma_{p, \mathrm{BNS}} \approx \frac{e N_{b}}{4}\left(2 \sigma_{z}\right) W_{\iota} \beta_{y}^{2},
$$

where $\Delta E$ and the betatron function are specified at the full energy of the linac. Solving for $\sigma_{p}$ and substituting in Eqs. (27) and (28) yields the alignment tolerance requirements and the lengtls of the correction section, respectively. If one uses Eq. (34) in Eq. (30), it is apparent that BNS damping will be insufficient to control multi-bunch transverse wakes in multi-GeV flavor factories. This technique may, however, be sufficient for several pulses in short $(\approx 1-\mathrm{GeV})$ linacs suitable for early linear light sources.

\subsection{Longitudinal Wakes and Beam Loading}

As the beam particles move through the waveguide structure they will lose energy into the structure. For the disk-loaded structure this loss cin be considered a difiraction loss by a plane wave witl the same power spectrum and Poynting vector at the iris radius as the actual field due to tlue clarge. The wake potential due to 
this process can be calculated using the same procedures as are used to compuse transverse wakes; again as niany modes as possible should be included. Fig. $(3 \mathrm{~b})^{1}$ shows the results of such a calculation for the SLAC structure. The importance of the higher order modes can be seeñ by the comparison of the total wake with that due to the excitation of the fundamental accelerating mode. (Note that a positive potential is decelerating.)

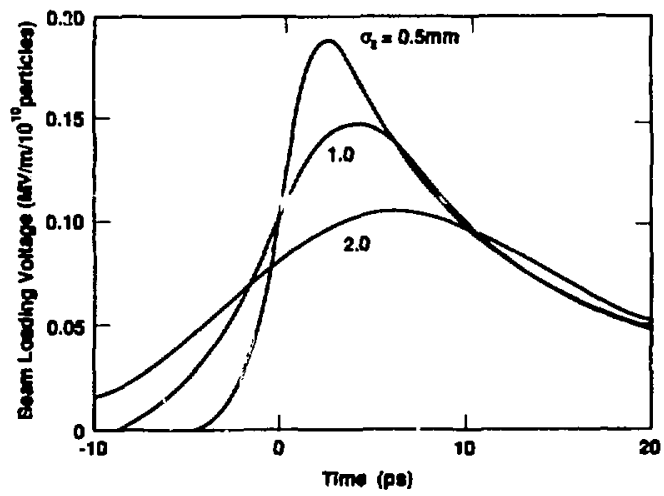

Figure 4. Beam loading voltage within a Gaussian bunch for the SLAC structure for three values of bunch length (adopted from Ref. 1).

The beam load" $\mathrm{g}$ potential is the convolution integral of the wake potential with the beam current. The value of the beam loading voltage, $E_{b}(t)$, within a Gaussian bunch (centered at $t=0$ ) is shown in Fig. 4 for bunches of three different lengths. The total energy gain of a particle at time $t$ in the bunch is the sum of the accelerating and beam loading voltages:

$$
E(t)=E_{a} \cos (\omega t-\theta)-E_{b}(t),
$$

where $\theta$ is the phase angle by which the center of the bunch leads the crest of the If accelerating wave. Both components of the energy gain give rise to an energy spread within a bunch of figite length. To some extent this spread can be reduced 

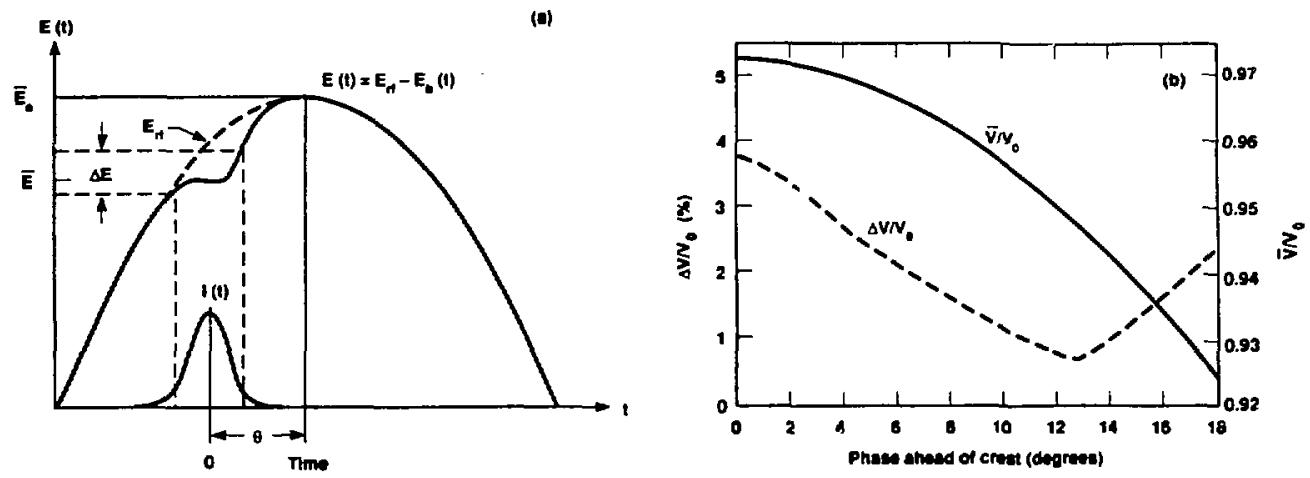

Figure 5. (a) Relationship between single-bunch beam loading $E_{b}(t)$, if accelerating wave $E_{r f}$, and net gradient $E(t)$; (b) energy spread and average gain per particle for the SLAC structure with $E_{a}=17 \mathrm{MV} / \mathrm{m}, N_{b}=5 \times 10^{10}$, and $\sigma_{x}=1.0 \mathrm{~mm}$ (adopted from Ref. 1).

by choosing the $\mathrm{q}$ so that the negative-going beam loading balances the increasing accelerating voltage. This effect is illustrated schematically in Fig. 5(a) and quantified in Fig. 5(b). The penalty for reducing the energy spread in this manner is reducing the average value of the energy gain of the pulse, $\langle E\rangle$, by a small amount.

As the acceptable energy spread of the beam is restricted (Sec. 1.) by FEL physics constraints (or luminosity constraints and energy resolution in flavor factories, one must be able to estimate how the wakefields affect $\Delta r$. One begins with an estimate ${ }^{3}$ of the longitudiral wake that depends on $\mathrm{rf}$ wavelength and iris aperture, a. For very short bunches, that is, for $\zeta \ll a$,

$$
{ }_{1} W_{1}(\zeta)=1.78 \cdot 10^{10} \frac{\mathrm{V}}{(\mathrm{C}-\mathrm{m})} a^{-2} .
$$

For $\zeta \approx a$,

$$
\begin{aligned}
{ }_{2} W_{1}(\zeta)=1.25 \cdot 10^{10} & \frac{\mathrm{V}}{(\mathrm{C}-\mathrm{m})} \zeta^{-1 / 2} a^{-1} \lambda^{-1 / 2} \\
& -24-
\end{aligned}
$$


For intermediate values of $\zeta$,

$$
W_{1}(\zeta)=\left[W_{1}^{-3}(\zeta)+{ }_{2} W_{1}^{-3}(\zeta)\right]^{-1 / 3}
$$

Next, following Palmer, one notes four distinct contributions to $\sigma_{p}$ : (1) the average energy of the bunch is reduced (zeroth order); (2) the tail of the pulse bas a greater loss than the head of the pulse (first order); (3) long pulses have a significant second order term with the rate of change of momentum falling at the tail; (4) Gaussian bunches have a significant third order term. To account for each of these contributions in computing the beam loading, Palmer divides the bunch into four equal sub-bunches: two at $\sim 0.2 \sigma_{z}$ and two at $\sim 1.4 \sigma_{z}$. The energy losses of the four bunches are

$$
\begin{aligned}
& V_{1}=M W_{l}(0), \\
& V_{2}=M\left[W_{l}(0)+W_{l}\left(1.2 \sigma_{x}\right)\right], \\
& V_{3}=M\left[W_{l}(0)+W_{l}\left(0.4 \sigma_{z}\right)+W\left(1.6 \sigma_{z}\right)\right], \\
& V_{4}=M\left[W_{l}(0)+W_{l}\left(1.2 \sigma_{z}\right)+W\left(1.6 \sigma_{x}\right)+W_{l}\left(2.8 \sigma_{z}\right)\right],
\end{aligned}
$$

where

$$
M=\frac{e N_{b}}{4 E_{a}} .
$$

From these beam loading voltages, Palmer then computes the first, second, and third order momentum spreads in the four-bunch approximation:

$$
\begin{aligned}
& { }_{1} \sigma_{p}=0.05\left(V_{3}-V_{2}\right)+0.35\left(V_{4}-V_{1}\right) \\
& { }_{2} \sigma_{p}=0.25\left(V_{3}+V_{2}\right)+0.25\left(V_{4}+V_{1}\right) \\
& { }_{3} \sigma_{p}=0.22\left(V_{3}-V_{2}\right)+0.033\left(V_{4}-V_{1}\right)
\end{aligned}
$$

A continuous pulse convolution integral gives more accurate results, but the fourbunch approximation should be sufficient for parameter surveys. 
As noted above, the first order effect can be canceled by the if with an appropriate choice of $\theta$. Since some of this momentum spread is required for BNS damping of the transverse wake, one should choose

$$
\tan \theta=\frac{1}{2 \pi} \frac{\lambda}{\sigma_{z}}\left({ }_{1} \sigma_{p}-\sigma_{p, \mathrm{BNS}}\right),
$$

where $\sigma_{p, \text { BNS }}$ is given by Eq. (36). Recall that $\sigma_{p, \mathrm{BNS}}$ is removed at the end of the linac. Including the effects of the of accelerating field, the total second order spread is

$$
{ }_{2} \sigma_{p, \mathrm{Lot}}={ }_{2} \sigma_{p}-0.5\left(\frac{2 \pi \sigma_{z}}{\lambda}\right)^{2} .
$$

For the purpose of designing the beam matching sections into th z wiggler, one can use as the final momentum spread

$$
\sigma_{p}=\left({ }_{2} \sigma_{p, \mathrm{tol}}^{2}+{ }_{3} \sigma_{p, \mathrm{Lol}}^{2}\right)^{1 / 2}
$$

5.2.1. Beam Efficiency. A quantity of considerable interest to the designer of linear colliders is the efficiency, $\eta_{b}$, with which the beam extracts energy from the rf structure:

$$
\eta_{b}=e s N_{b} \frac{<E \geq}{E_{a}^{2}} \approx \frac{e s N_{b}}{E_{a}} \propto \frac{N_{b}}{\lambda^{2}},
$$

where the uncorrected elastance $s$ is given by Eq. (2.9b). From Eq. (37) $<E>$ differs from $E_{a}$ by the beam loading voltase, $E_{b}$. An approximation to Eq. (48) for structures with the ratio $a / \lambda$ roughly the same as the SLAC structure is

$$
\eta_{b} \approx 0.13 \%\left(\frac{N_{b}}{10^{10}}\right)\left(\frac{100 \mathrm{~mm}}{\lambda}\right)^{2}\left(\frac{100 \mathrm{MeV} / \mathrm{m}}{E_{a}}\right) .
$$

The efficiency of transferring energy from the of source to the beam is the product of the beam efficiency and the structure efficiency, i.e., $h, \eta_{b}$.

A XRFEL or collider macro-pulse will contain $B$ bunches of equal charge. In order that each bunch extract the same fraction of the the stored encrgy from the 


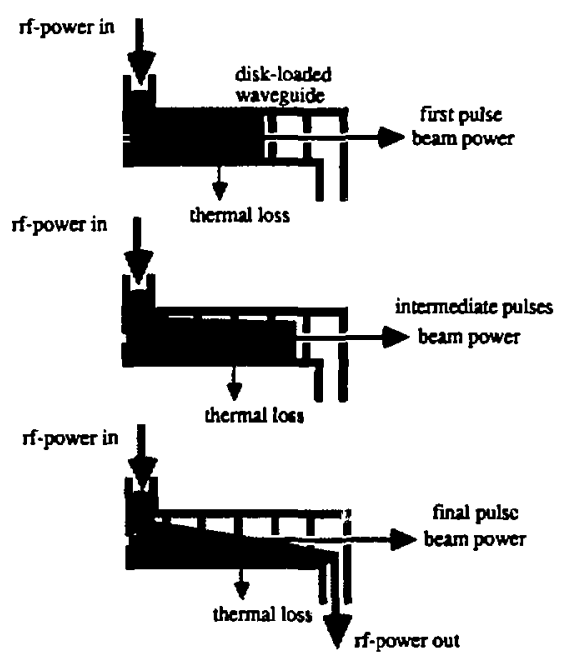

Figure 6. Efficient energy extraction from a travelling wave linac section for short pulse trains

structure, the beam loading must be compensated for in one of several possible ways (Fig. 6). For example, the first pulse can be injected before the section is completely filled, and the bunch-to-bunch spacing and power flow can be adjusted so that the energy added between bunches just compensates for the pulse-to-pulse sag.

An alternative procedure suitable for use with pulse trains of duration much greater than $T_{f i l l}$ is to recirculate the rf power that exits from a section by adding it back at the input of the same section. A steady-state condition cin be achieved after $\sim 3-5 T_{f i l l}$, at which point the beam is injected.

Although both scliemes of compensating for beam loading will have the side effect of reducing the gradient of the linac, they allow one to raise the overall 
electrical efficiency of the accelerator to

$$
\eta_{\mathrm{lol}}=h_{s} \eta_{b} \eta_{r f}\left(\frac{\dot{B} T_{\mathrm{flll}}}{T_{r f}}\right)
$$

where $\eta_{r f}$ is the efficiency of converting electrical power to rf and $T_{r f}$ is the total period during which if is supplied to the high gradient structure. With both efficient If power sources and beam-loading compensation it should be practical to obtain $\eta_{\text {tot }} \approx 10 \%$.

From the analysis of the previous section one should note that increasing $N_{b}$ to increase the bcam efficiency is limited by wake field effects. Via longitudinal wakefields raising $N_{b}$ will lead to the sinultaneous introduction of an irreducible, nonlinear momentum spread in the bearı. Raising the beam loading has the additional drawback of increasing the amount of energy spread needed to BNS damp the single bunch transverse wakefield. The length of the section designed to remove this energy spread will, therefore, increase with increased beam loading. Fortunately, however, in accelerators for linear light scurces this increase in overall linac length is a $1-2 \%$ effect.

\section{PULSE MANIPULATION}

Lincar light sources will probably have several output beamlines. In addition to directing the beam to the appropriate beamline, the final beam manipulation subsystem may be required to perform other functions:

1) Stretch the length of the bunch from its value in the high frequency, high gradient accelerator to a much longer pulse matclied to user requirements.

2) Focus the beam from its $\approx 100 \mu \mathrm{m}$ sizc in the accelerator to the $\approx 1 \mu \mathrm{m}$ sizc appropriate to self-focuscd plasma wigglers or other plasma experiments.

3) Provide for a vacuum-to-gas transition in tlec casc of plasma devices. 


\subsection{Pulse Stretching}

The stretching of the pulse can be accomplished by taking advantage of the irreducible momentum spread acquired during the acceleration process. Although many forms of dispersive transport may be used as a debuncher, for scaling purposes it is convenient to choose the four dipole chicane arrangement (Fig. 7), typically used as a debuncher in conjunction with damping rings.

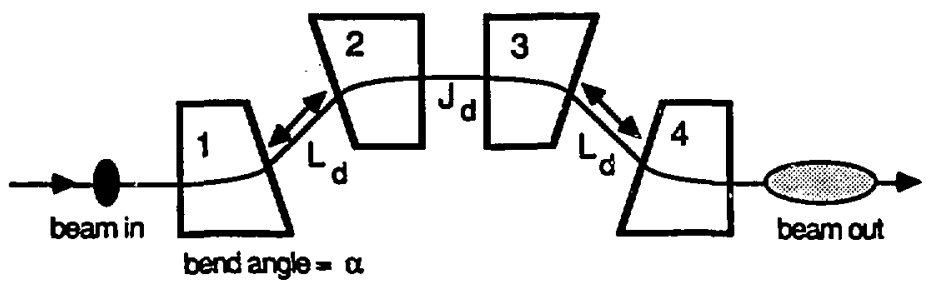

Figure 7. Four dipole pulse stretcher in a chicane arrangement

Dipoles 1 and 2 and dipoles 3 and 4 are separated by $L_{d}$ meters; the distance between dipoles 2 and 3 is $J_{d}$ meters. With four dipoles of equal strength and with the beam trajectory normal to the entrance and exit edges of the dipoles, the chicane can be made doubly achromatic to second order. The effect of the chicane is described $^{18}$ by a transfer matrix, $M$, with unit diagonal elements and two nonzero off-diagonal terms. The dispersion of the chicane is described by a debunching parameter (in units of $\mathrm{cm} / \% \Delta p / p$ ):

$$
M_{34}=2 L \tan ^{2} \alpha+4 \rho \tan \alpha+4 \rho \tan \alpha-8 \rho \sin \alpha,
$$

where $\alpha$ is the angle of bend and $\rho$ is the radius of curvature in the magnetic field. The only other nonzero matrix relates the entrance position to the exit angle; that is

$$
M_{12}=4 \rho \tan \alpha+\frac{2 L}{\cos ^{2} \alpha}+J_{d} .
$$


The magnetic field and the radius of curvature are related by

$$
B \rho=33 \mathrm{~T}-\mathrm{m}\left(\frac{E}{10 \mathrm{GeV}}\right)
$$

Precision, small aperture ( 4 to $5 \mathrm{~cm}$ ) dipoles are practical with a field strength of $6.5 \mathrm{~T}$. With such magnets, pulse stretcling to $\approx 1 \mathrm{~cm}$ with $\Delta p / p= \pm 1 \%$ is achievable in a clicane of overall length less than $7 \mathrm{~m}$. Thus, compact linear light sources can tailor the duration of the pulse up to that available with circular synchrotron sources.

The drawback to stretcling the pulse by more than a factor of several is that the peak current in the linac is raised to more than $1 \mathrm{kA}$. Although increasing the peak current does not lead to a critical constraint in the linac proper, it does make the task of designing the low emittance injector ever more difficult. In tradeoff studies it is probably unwise to drive peak current from the injector much above $1 \mathrm{kA}$.

\subsection{Beam Matching}

The matching section for a flavor factory or a plasma-focused wiggler XRFEL system is analogous to the: final focts of a linear collider flavor factory and scales accordingly. In the final focus the beam radius must be reduced by a fnctor $\approx 100$ in as short a distance as possible; i.e., the design must have the minimum achievable $\beta^{*}$. Let $\beta_{0}^{*}$ be the minimum that can be aclieved for a given momentum spread by a focal array with no cliromatic correction. Chromatic correction elements can reduce this value by a factor $S$ which scales inversely with $\sigma_{p}$. Thus,

$$
\beta^{*} \geq \frac{\beta_{o}^{*}}{S}=\frac{\beta_{o}^{*} \sigma_{p}}{S_{o}}=25 \beta_{o}^{*} \sigma_{p}
$$

Unlike the casse of the linear collider, the focal spot for a plasma focused XRFEL may not be lighly clliptical; therefore, quadrujesle cloublet elesigns are ruled out. 
An appropriate design for scaling is a quadrupole triplet with a le sut (Fig. 8) based on the SLC final focus, which brings the beam to a circular focus. One scales such a design by modifying all lengths by one factor and all transverse dimensions by another. The scaling factors are respectively $f^{*}$, the "ideal" focal length, and $a^{*}$, the aperture radius of the first quadrupole, which has pole tips of field strength $B^{*}$. The "ideal" focal length is

$$
f^{*}=\left[\left(\frac{a^{*}}{B^{*}}\right)(B \rho)\right]^{1 / 2},
$$

where $B \rho$ is given by Eq. (53). Palmer ${ }^{3}$ expresses the performance of the focal system in terms of $f^{*}, a^{*}$ and constants that depend on details of the magnet system: $T, A_{x}, A_{y}$ and $\Lambda$.

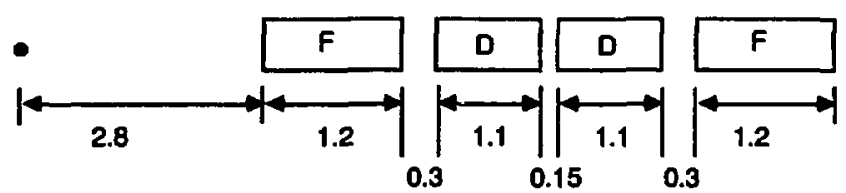

Figure 8. Quadrupole triplet array for beam matching based on the SLC design. All quadrupoles have a field gradient of $50 \mathrm{~T} / \mathrm{m}$. Distances are given in meters.

In terms of the scaling parameters, the $\beta^{*}$ of the focal array is

$$
\beta_{o}^{*}=2 \sigma_{p} f^{*} T
$$

The maximum angular acceptance of the focal array and the free space before the first quadrupole are

$$
\Theta_{x, y}=\frac{a^{*}}{A_{x, y} f^{*}}
$$

and

$$
\begin{gathered}
l_{1}=\Lambda f^{*} \\
-31-
\end{gathered}
$$


respectively. Combining Eqs. (54), (55) and (56), one obtains

$$
\beta^{*}=\left(\frac{T}{S_{0}}\right) \beta \rho\left(\frac{a^{*}}{f^{*} B^{*}}\right) \sigma_{p}^{2} .
$$

For this design, $T=2.96, A_{x}=4.3, A_{y}=3.2, \Lambda=1.36$, and $S_{0}=0.04$.

In order to focus the beam to the very small radii, the pole tip fields must either be very strong or the aperture must be very small. Electro-mechanical engineering considerations are likely to limit the minimum aperture of superconducting quadrupoles to $2-3 \mathrm{~cm}$. In that case the field strength will have to be raised to $>6 \mathrm{~T}$. The ali. 'tive is to choose a conventional magnet design with iron pole pieces. For this case the pole tip fields will be limited to $\approx 1.5 \mathrm{~T}$. More than compensating for this limitation is the fact that the field can be led into a very small region of space (apertures $\approx 1-2 \mathrm{~mm}$ ) at the expense of magnet efficiency. Therefore, the system designer should opt to use conventional magnets, even though cryogenic systems will be almost mandatory in the design of a pulse stretcher (if that is desired).

\subsection{Gas Matcling for Self-Focused XRFELs}

If the size of the focal spot is not equal to the self-focused radius of the beam in gas, the equilibrium beam size will differ from the size of the focal spot. This consideration is especially important in the case that the vacuum-air transition is a very thin foil rather than a differentially pumped transition. Foils may be useful in bringing the accelerator to early operational status at low repetition rates.

If the focal system produces a spot of radius $a_{f}$, then in the gas the beam will suffer radial oscillations, eventually settling to an equilibrium radius given by

$$
a_{c q}^{2}=a_{f}^{2} \exp \left(\theta_{\mathrm{tot}}^{2} \frac{\gamma I_{o}}{I_{b}}-i\right),
$$

where $I_{0}=17 \mathrm{kA}$. The mean betatron angle, $\theta_{101}$, is determined by a combination of the emittance and the foil induced scattering:

$$
\begin{gathered}
\theta_{\mathrm{lot}}^{2}=\left(\frac{\varepsilon_{n}}{\gamma a_{f}}\right)^{2}+\theta_{\mathrm{scat}}^{2} . \\
-32-
\end{gathered}
$$


For very thin foils of thickness $X$, the Moliere-corrected rms scattering angle is

$$
\theta_{\mathrm{Bcat}}=\left(\frac{28}{\gamma}\right)\left(\frac{X}{X_{\mathrm{rad}}}\right)^{1 / 2}\left[1+0.111 \log _{10}\left(\frac{X}{X_{\mathrm{rad}}}\right)\right],
$$

where $X_{\text {rad }}$ is the radiation length of the foil material or of the gas in the transition region in the case of a plasma assisted XRFEL.

\section{CHOICE OF FREQUENCY AND RF POWER SOURCES}

The generation of bright beams at multi-GeV energies requires the accelerator designer to consider new classes of machines that can operate with gradients exceeding $200 \mathrm{MeV} / \mathrm{m}$ in structures suitable for high-repetition rate-operation. The approach must be highly reliable and energy efficient, if the electrical power demands of accelerator operation are to remain within currently accepted bounds for most facilities. As discussed in the Sec. 3, the accelerating gradient can be increased and the energy required per meter of accelerator structure reduced by scaling the (effective) If structure of the accelerator to high frequencies ( $\geq 10 \mathrm{GHz}$.) The practical limits of this approach for conventional of cavities are set by the electron induced breakdown limit and the surface heating limit, with little gain in gradient being aclievable for frequencies exceeding $30 \mathrm{GHz}$. Considering that both the cost of fabricating the of structure and the difficulty of maintaining the alignment of the structure is likely to increase once the cavity is miniaturized beyond a scalc size of $\approx 2 \mathrm{~cm}$, one can select $10-20 \mathrm{GH} z$ as the best frequency range for a compact accelerator. If standing-wave linacs with geometries more complex than that of the disk-loaded waveguide are used to accelerate long pulse trains, ease of fabrication will tip the balance even further in the direction of choosing longer if wavelengths.

The peak of power needed to drive an accelerator based on a generic $2 \pi / 3$ diskloaded waveguide can be detcmined by substituting Eqs. (6)-(13) into Eq. (16). A useful, though less exact, estimate can be olstinined by ignoring the dependence 
of the elastance on the group velocity. Scaling the SLC linac design at constant $\beta_{g}$-that is, constant $a / \lambda$ - one can rewrite $\mathrm{Eq}$. (16) as

$$
P_{r f}=74 \mathrm{MW} / \mathrm{m}\left(\frac{E_{a}}{100 \mathrm{McV} / \mathrm{m}}\right)^{2}(h \tau)^{-1}\left(\frac{\lambda}{105 \mathrm{~mm}}\right)^{1 / 2} .
$$

For the SLC design $h \tau$, the product of the structure efficiency and attenuation parameter is $\approx 0.3$; halving the fill time will reduce $h \tau$ to $\approx 0.2$. Thus, a rough estimate of the total peak power needed to drive a accelerator is

$$
P_{\mathrm{rf}} \approx 60 \mathrm{GW}\left(\frac{E}{10 \mathrm{GeV}}\right)\left(\frac{\lambda}{105 \mathrm{~mm}}\right)^{1 / 2} .
$$

One concludes that a new class of $\mathrm{ff}$ power sources will be needed to drive compact accelerators of beams for compact linear light sources and flavor factories.

\subsection{Relativistic Ḱlystrons}

The high energy accelerator community has considered many approaches to providing the large amounts of $\mathrm{rf}$ power at $\mathrm{X}$-band and higher frequencies necded to achieve ultra-high accelerating gradients. Recently a consensus ${ }^{13}$ has emerged in the U.S., Western Europe, and the USSR that the range from 10 to $30 \mathrm{GHz}$ holds the most promise for the next generation $\mathrm{TcV}$ linear collider. The same considerations apply to the accelerator for a compact linear light source. At the low end of this frequency range one can perhaps combine many conventional klystrons with an advanced if pulse-compression network to power the accelerator. More novel approaches, however, seem to offer the required performance levels at stibstantially lower cost and complexity. One of these approaches, the relativistic klystron, offers particular promise, especially if high repetition rates are desired as in the case of flavor factories. The relativistic klystrom ${ }^{20}$ (Fig. 9) is a concept for modulating a multi-kA, multi-MeV beam produced with an lincar induction accelerator (LIA) at the desired of frequency (10 to $15 \mathrm{GH} z$ ) and using the modulated high current bean to excite an if generating transfer stuncture. The high poik power of is then fed via 


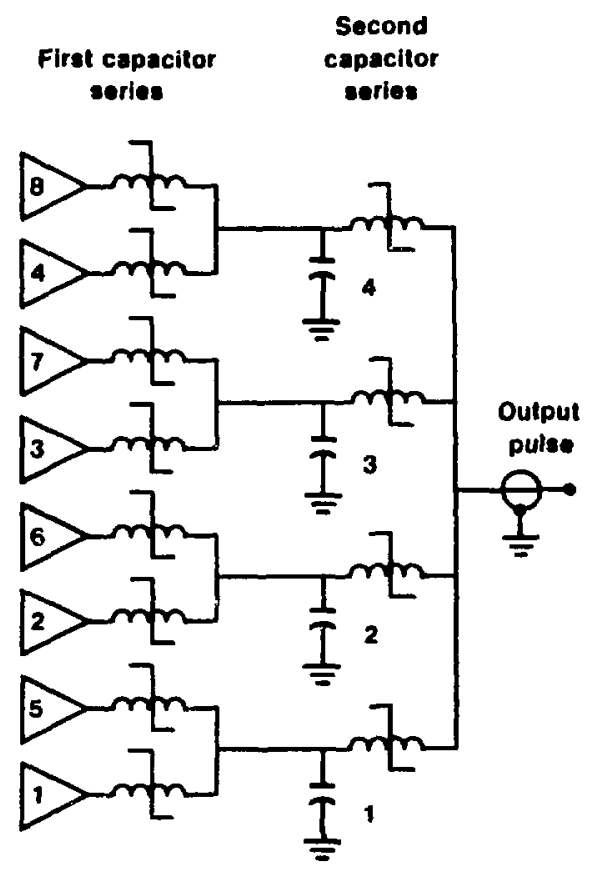

Figure 9. Conceptual design of the front end of a relativistic klystron powered by a multi-kA, multi-MeV beam from an induction linac.

waveguides to the miniaturized If cavities of the high gradient $(200-400 \mathrm{MeV} / \mathrm{m})$ accelerator of the high energy beam.

Three basic variants of the relativistic klystron concept are now under active investigation by a SLAC/LLNL/LBL collaboration. These are:

1) Extrapolation of conventional (velocity modulation), high gain klystron design to $1-3 \mathrm{MV}$ operation $(\approx 1 \mathrm{GW})$.

2) Adiabatic deceleration of an energy modulated, bunched, 5-10 MeV beam with multiple klystron output cavities $(\approx 10 \mathrm{GW})$.

3) Alternating multiple cavity deceleration with induction re-acceleration of the high current beam to form a single power "tube" ( $\approx 100 \mathrm{GW})$. 
Early experiments ${ }^{21}$ at LLNL have already yielded record power levels $(\approx 80 \mathrm{MW})$ for X-band klystrons at high conversion efficiency $(\approx 55 \%)$. Over $200 \mathrm{MW}$ have been produced at higher frequency $(11.4 \mathrm{GHz})$ although at lower efficiency. This power was sufficient to yield an accelcrating field of $130 \mathrm{MeV} / \mathrm{m}$ in a small section of high gradient structure. The most convincing demonstration of the practicality of the relativistic klystron approach would be the construction and operation at the carliest possible date of an ultra-compact (5-m), 1-GeV linac powered by the beam from the 7-MeV, 3-kA ETA-II at LLNL.

Before such an ambitious goal can be come a reality, the collaboration must address several fundamental plysics issues relating to (1) If generation and power handling and (2) stability of the drive beam in the of generating structures. Still, for the purposes of scoping studies, the design space for the induction linac beam source can already be roughly delimited:

1) Both the acceleration and deceleration rates for the high current beam can exceed $1 \mathrm{MeV} / \mathrm{m}$.

2) Using present design techniques, the maximum if current that can be handled efficiently with standing wave klystron cavities is $3-4 \mathrm{kA}$.

3) The minimum voltage beam that can be efficiently decelerated in a multi-cavity transfer structure is $2-3 \mathrm{McV}$.

4) Maximum beam-to-rf conversion efficiencies of 50-70\% seem possible.

5) The efficiency of converting electrical energy into usable beam encrgy with induction linacs is $\approx 50 \%$.

Thus, a flavor factory using the relativistic klystron approach would requirc $\approx 60 \mathrm{MeV}$ of LIA cells operating with a 2-kA beam to provide sufficient power to drive two $6-\mathrm{GeV}$, compact if linacs operating at $\approx 11 \mathrm{GHz}$. 
The large power flow earried by LIA beams is not the scle reason for their interest to the high energy accelerator designer. Because the magnitude of the transverse wake varies as $a^{-3}$, one is unlikely to scale the disk-loaded structure to $\mathrm{X}$-band or higher frequencies while maintaining $A_{l} \equiv a / \lambda$ constant. Rather one should increase $A_{l}$ by a factor of 2 to 3 to control wakeficlds. Increasing $A_{l}$ has several additional effects. First, by Eq. (7), raising $A_{l}$ will increase the if gro"s velocity thereby shortening the fill time of the structure. The if attenuation time of the structire also varies as $\lambda^{3 / 2}$. Therefore, scaling with constant attenuation parameter $t$ implies shortening the fill time from the $\approx 1 \mu \mathrm{s}$ a: SLC to $\approx 50 \mathrm{~ns}$ for an $\mathrm{X}$-band linac.

These short fill times match the pulse durations of modern induction linacs. The second effect of increasing $A_{l}$ is to raise the maximum surface field that is associated with a given accelerating field. As long as sufficient if power is available, one will probably choose to operate the linac at as close to the maximum surface ficld as is practical. Therefore, shortening the fill time by opening the iris aperture comes at the expense of reducing gradient.

With respect to the design of its rf power source, a linear light source could have one significant difference from the lirear collider. The collider's power source must deliver of for approximately one fill time. In contrast, some applications of compact linacs may require lundreds of bunches in a macro pulse. In that case, the If power must be supplied for a time, $T_{r f}$, several times longer than the fill time; i.c., $\approx 300-1000 \mathrm{~ns}$.

Moden induction linacs with ferrite cores produce pulses with a duration of several tens of nanoseconds. From the design principles described in Ref. 22, one sees that scaling ferritc loaded LIAs to produce continuous pulses much longer than $\approx 100 \mathrm{~ns}$ is probably impracticul. By changing the ferromagnetic material to $\mathrm{n}$ 
dielectric coated metallic glass, the pulse duration may be extendible to $\approx 200$ 300 ns. However, the beam break-up effects of this change are not known. The first induction linacs such as the Astron with soft iron tape cores produced 300 ns pulses. Indeed, a 1- $\mu$ s pulse core was built at NBS in the early 1970s. At $1 \mu$ s such cores are hardly small or incxpensive. Moreover, eddy current losses in the metallic cores will be high. The alternative is to recycle the induction cores at multi- $\mathrm{MHz}$ rates with branched magnetic drives. ${ }^{23}$

7.1.1. Branched Magnetic Drives. The magnetic pulse compressors that supply pulsed power in modern LIAs consist of a repetitive pulse source followed by several serial stages of pulse compression. These networks can drive the LIA at any repetition rate up to the maximum firing rate of the primary commutator. In the ETA-II, for example, this rate is set by the recovery time of the thyratron switches. If the applicaticn were to require a fixed number (3-10) of output pulses from the LIA in a burst (1-2 $\mu \mathrm{s})$, a new scheme would become feasible. The technique, called branched magnetics, involves deploying several capacitive stores and switches in parallel that are fired in a sequence at any repetition rate permitted by the load. One such circuit was tested with an eight-pulse burst (Fig. 10). This proof-of-principle experiment demonstrated pulse generation into a resistive load at $16 \mathrm{MHz}$.

In the application of this approach to synthesize microsecond bursts of LIA beam power, the firing rate will be linited by the time required to reset the induction cores in the linac. Unless the reset times are at least twice as long as the original pulse time of the core, the amount of power consumed by the reset circuits will be comparable to that of the beam drive circuits. Hernce, the highest practical duty factor of the LIA during the macro-pulse is $\approx 30 \%$. Therefore, even with brauched magnetics, supplying of power for $\approx 1 \mu \mathrm{s}$ will eitluer reçuire multiple LIAs or an innovation in of power handling. One such idea is recycling the if power that leaves 


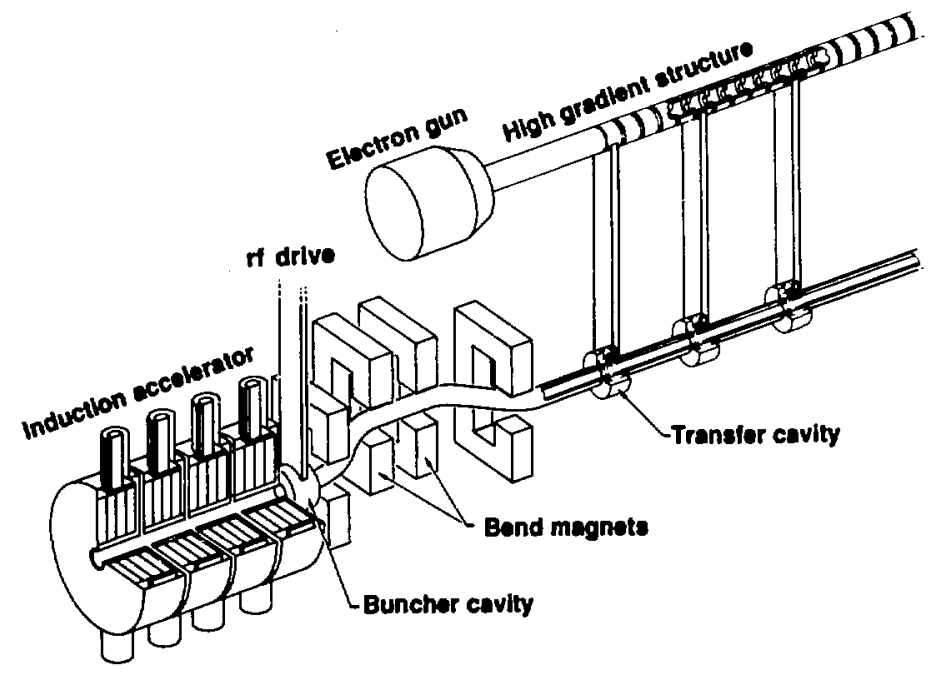

Figure 10. Simplified cicuit diagram of the burst mode generator designed to provide any desired repetition rate for a fixed number of pulses (in this case, eight). The numbers indicate the order in which the capacitors are discharged. one linac section into another sectiori. Maintaining adequate if phase control and stability at high power is a critical physics issue for this latter approach.

From the point of view of making the LIA beam source as simple and economical as possible, the accelerator designer should endevour to make $T_{r f}$ as small as possible. In particular, the fill time must be kept below $100 \mathrm{~ns}$ without making $L_{f}$ so short that both the peak power and number of if feeds are raised excessiveiy. Therefore, even if the size (gradient) constraints were eased considerably, the accelerator designer would be strongly motivated to build the linac at X-band or higher frequency. 


\section{COST AND SIZE ESTIMATES}

The primary capital cost element of the accelerator will be the LIA driven, relativistic klystron power sources for the of linac. The considerations that govern the installed capital cost of such of sources is discussed at length in Ref. 22 for the case of single branch magnetic drives. It remains only to indicate how to modify those scaling relations to the case of a an induction drive accelerator with branched magnetics.

One begins by determining the total active length of high gradient accelerator, including the section to correct energy spread:

$$
L_{\text {act }}=50 \mathrm{~m}\left(\frac{E}{10 \mathrm{GeV}}\right)\left(\frac{E_{a}}{200 \mathrm{MeV} / \mathrm{m}}\right)+L_{\text {corr }} .
$$

The overall length of the linac is then

$$
L_{\mathrm{acc}}=\left(1+F_{q}\right) L_{\mathrm{act}} .
$$

Using Eq. (16), one then calculates the total voltage to be supplied by the induction cells as

$$
V_{\text {ind }}=\frac{\Pi_{r f}}{I_{\text {ind }} \eta_{\mathrm{rr}}}=10^{-3} P_{\mathrm{rr}} L_{\text {acc }}\left(\frac{2 k \mathrm{~A}}{I_{\mathrm{ind}}}\right)\left(\frac{0.5}{\eta_{\mathrm{rf}}}\right)
$$

where current in the LIA is $I_{\text {ind }}$. The LIA pulse length, $T_{\text {ind }}$, the number of LIAs, $N_{\text {ind }}$, and the total number of magnetic drive branches in all LIAs, $N_{\mathrm{br}}$, depends on the maximum pulse length, $T_{\text {inax }}$, assumed for the induction cores and on the induction reset duty factor during the macro-pulse, $\Delta_{\text {ind }}$. In ternıs of the standard number theoretic functions,

$$
\begin{aligned}
N_{\text {ind }} & =\operatorname{Min}\left[\left(\operatorname{Int}\left(\frac{T_{\mathrm{rf}}}{T_{\text {imax }}}\right)+1\right),\left(\operatorname{lnt}\left(\Delta_{\text {ind }}^{-1}\right)+1\right)\right] ; \\
N_{\mathrm{br}} & =\operatorname{Int}\left(\frac{T_{\mathrm{rl}}}{T_{\mathrm{inax}}}\right)+1 ; \\
T_{\text {ind }} & =\frac{T_{\mathrm{rl}}}{N_{\mathrm{br}}} .
\end{aligned}
$$


Once these quantitics are determined the cost scaling algorithm can be applied with the modifications indicated in the Appendix. For quick estimates of the cost of powering the one can use the graph of rf cost vs total "tube" power given in Fig. 11.

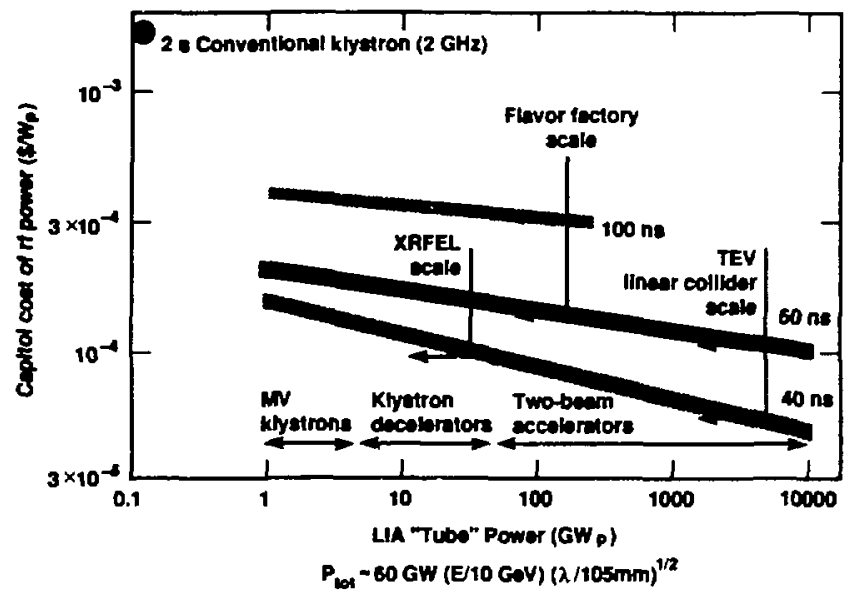

Figure 11. Rf cost vs. "tube" power for various LIA pulse lengths

With respect to scaling the cost per active meter of the disk-loaded waveguide, actual data at many frequencies are scarce. The following heuristic equation seems, however, to summarize the experience of the accelerator community:

$$
C_{\text {wave }}=45 \mathrm{k} \$ / \mathrm{m}\left(\frac{\lambda}{500 \mathrm{mmn}}\right)^{1 / 3}+2.5 \mathrm{k} \$ / \mathrm{m}\left(\frac{50 \mathrm{~mm}}{\lambda}\right)^{5 / 2}+5 \mathrm{k} \$ / \mathrm{m} \text {. }
$$

The first term, representing a cost of materials and machining, scales as the waveguide volume. The second term is an alignment cost, which scales as the transverse walie. The final tern is a fixed handling cost.

Klystron transfer cavities and power feeds to the high gradient structure are estimated to cost

$$
C_{\text {cav }}=20 \mathrm{l} \$ / \mathrm{m}
$$


The final bearn manipulation beamline is estimated to cost

$$
C_{\mathrm{bmln}}=200 \mathrm{k} \$ / \mathrm{nn}
$$

As is done for the cost of the rf power, the costs of Eqs. (71), (72), and (73) should be multiplied by $\approx 1.35$ to account for the associated manpower needed for installment, procurement, and project managenent.

\section{ACKNOWLEDGMENT}

The plyysics reported here has been borrowed liberally from many sources. With respect to the design of high gradient rf accelerators, the work of Robert Palmer (SLAC and BNL) and Peity Wilson (SLAC) in analyzing linear colliders has provided indispensible guidance. Andrew Sessler (LBL) has been an effusive source of ideas concerning the use induction technology to power high gradient accelerators. Dan Birx (Science Research Laboratory) has provided valued assistance in extrapolating induction linacs into the realm of branched magnetics and hitherto unexplored cavity geometries. Others from LLNL who have contributed importantly include Willium Fawley, Kelvin Neil, and Art Paul. 
Appendix A. COST SCALING ALGORITHM FOR BRANCHED DRIVES

Variables used in the cost scaling Eqs. (22) for the induction driver with branched magnetics include repetition frequency $f$ in $\mathrm{Hz}$, the pulse length (fullwidth half maximum), the total accelerating voltage $V$ in $M V$, the total volt-seconds of ferrimagnetic core $W=V T_{\text {ind }}$, the peak gap stress $E_{g}$ in $\mathrm{kV} / \mathrm{cm}$, the single pulse energy $E=V I_{\text {beem }} T_{\text {ind }}$ in joules, the average power $P=f E$ in $M W$, the effective gradient $G$ in $\mathrm{MV} / \mathrm{m}$, and the inner radius of the ferrites in the induction cells, $r_{2}$, in $\mathrm{cm}$ which is taken as the beam pipe radius plus $2 \mathrm{~cm}$ to account for the thickness of the focusing solenoids. In the scaling equation (A1) italicized quantities refer to injector voltage, power, pulse energy, etc. Costs are specified in constant FY88 k\$. The branched magnetic drives are composed of $N_{b r}$ branches and $N_{i n d}$ induction linacs.

\section{A.1. Injector Subsystem}

The scaling equation for the injector is divided into five separate components:

$$
\begin{aligned}
C_{\text {inj }}= & \left\{545\left(\frac{W}{0.225}\right)_{\text {cells }}(I / 3000)+320\left(\frac{E}{630}\right)_{\text {mag }}+450\left(\frac{P}{2.25}\right)_{\text {isps }} \operatorname{Max}\left(1, \frac{f}{500}\right)\right. \\
& \left.+165\left(\frac{V}{3}\right)_{\text {vac }}^{3}+(60)_{\text {fixtures }}+60\left(\frac{I}{3000}\right)_{\text {callode }}^{1 / 2}\right\} .
\end{aligned}
$$

To provide a stiff voltage source for the injector the magnetic compressors provide $50 \%$ additional energy into resistive loads. As branclied drives may comprise as many as three separate induction linacs, the injector cost should be multiplied by $N_{\text {ind }}$ in the estimate of total laardware cost. 


\section{A.2 Beam Transport Subsystems}

For solenoidal transport, the cost has three separate components:

$$
\begin{aligned}
C_{\text {sol }}=\{[(57 & \left.\left.+40\left(\frac{r_{i}}{7.5}\right)\left(\frac{I}{3000}\right)\right)\left(\frac{V}{1.5}\right)\left(\frac{I}{3000}\right)\left(\frac{r_{i}}{7.5}\right)\left(\frac{0.75}{G}\right)\right]_{\text {Iocus }} \\
& \left.+\left[42\left(\frac{V}{1.5}\right)\left(\frac{r_{i}}{7.5}\right)\left(\frac{0.75}{G}\right)\right]_{\text {steer }}+[78]_{\text {match }}\right\} .
\end{aligned}
$$

For the alternative laser guiding scheme the scaling equation is

$$
C_{\text {laser }}=\left(\frac{7.5 V}{G}\right) N_{\text {ind }}
$$

which includes laser, gas handling, and matching magnet costs for $f \leq 250 \mathrm{~Hz}$.

\section{A.3. Accelerator Cell Subsystems}

The cell block cost is

$$
C_{\text {block }}=\left[150\left(\frac{V}{1.5}\right)\left(\frac{r_{\mathrm{o}}}{20}\right)^{2}\left(\frac{0.75}{G}\right)\left(\frac{175}{E_{g}}\right)\right] N_{\text {ind }},
$$

where $r_{0}$ is related to $r_{i}$ by

$$
r_{\mathrm{o}}=r_{i}+\left(\frac{G T_{\text {ind }}}{p}\right) \Delta B
$$

Here $p$ is the fernite packing fraction $\sim 0.8$ and $\Delta B$ is the total flux swing $~$ $0.6 \mathrm{~Wb} / \mathrm{m}^{2}(=180 \mathrm{MV} / \mathrm{m})$, and effective gradients range from 0.5 to $1.5 \mathrm{MeV} / \mathrm{m}$. The ferrite cost is given by

$$
C_{\text {ferrite }}=\left[140\left(\frac{W}{0.225}\right)\left(\frac{r_{o}+r_{1}}{27.5}\right)\right] N_{\text {ind }},
$$

where we have used the relation between the ferrite area $A$ and volunse $\pi A\left(r_{0}+r_{i}\right)$. The cost of intermediate stores and power supplics scales as

$$
C_{\mathrm{isp \textrm {M }}}=\left[678\left(\frac{P}{3.2}\right) \operatorname{Max}(1,500 / f)+5\left(\frac{E}{630}\right)\right] N_{\mathrm{br}} .
$$


If the repetition rate of the system is less than $600 \mathrm{~Hz}$, the power in Eqs. (A1) and (A7) should be replaced by a value corresponding to $600 \mathrm{~Hz}$ operation. The scaling for the magnetic pulse compressors is

$$
C_{\mathrm{mag}}=\left[241\left(\frac{E}{630}\right)\right] N_{\text {ind }} .
$$

For power sytems delivering pulses at repetition rates $\approx 100 \mathrm{~Hz}$ the magnetic modulators and intermediate stores can be re-engineered to reduce costs by nearly factor of two. The cost of the strongback alignment structure is

$$
C_{\text {strong }}=\left[450\left(\frac{V}{12.5}\right)\left(\frac{T_{0}}{20}\right)^{2}\left(\frac{0.75}{G}\right) K\right] N_{\text {ind }} .
$$

where the constant $K$ depends on the focusing scheme; namely,

$$
\begin{aligned}
K & =0.6 \text { for solenoids, } \\
& =0.5 \text { for laser guiding. }
\end{aligned}
$$

\section{A.4. Ancillary Subsystems}

The cost for low and extremely low conductivity water will scale as

$$
C_{i c w}=90\left(\frac{P}{13.1}\right) N_{b r},
$$

for $f>100 \mathrm{~Hz}$. For $f<100 \mathrm{~Hz}$ one should use the value of $C_{l \mathrm{c} w}$ at $f=100 \mathrm{~Hz}$. The vacuum system is scaled as if it were pumping speed limited (valid for $r_{i}<2.5 \mathrm{~cm}$ ):

$$
C_{\mathrm{vac}}=660\left(\frac{V}{12.5}\right)\left(\frac{r_{1}}{7.5}\right)^{2}\left(\frac{0.75}{G}\right) K N_{\text {ind }},
$$

where $K$ is the same as in $E_{1}$. (A3). The cost of electrichl fluids is proportional to average beam power;

$$
C_{\text {lluid }}=542\left(\frac{P}{13.1}\right) N_{b r}
$$

The scaling of dump costs is similar is that for reactors; i.e., $\$ 1$ per watt of time average beam power into the dump, $P_{d}$. For the relutivistic klystron assume that 
the average beam voltage at the dump, $V_{d}$, is $5 \mathrm{MeV}$; hence, $P_{d}=V_{d} T_{\text {ind }} I_{\text {beam }} f$. The cost of the dump is, therefore,

$$
C_{\text {dump }}=1000 P_{d} N_{\text {ind }} \text {. }
$$

The cost of fixtures is proportional to the length of the induction linac:

$$
C_{\text {fixture }}=20\left(\frac{V}{12.5}\right)\left(\frac{0.75}{G}\right) N_{\text {ind }} \text {. }
$$

The cost of the instruments and controls scales as a fixed (buy-in) value plus a percentage of the cost of the hardware to be monitored and controlled:

$$
C_{\text {idec }}=1500+0.04\left(C_{\mathrm{inj}}+C_{\mathrm{cell}}+C_{\mathrm{locus}}\right) \text {. }
$$

Summing the cost equations yields the total hardware cost for the induction linac driver.

\section{A.5. Installation and Engineering Support}

The installation costs for the base design were estimated for each component; for estimating purposes the installation costs can be taken as a fixed percentage of the total hardware costs adjusted to the fully loaded labor rate, $R$, in $\mathrm{k} \$ / \mathrm{man}$ month.

$$
C_{\text {install }}=0.09 C_{\text {hardware }}\left(\frac{R}{10}\right) .
$$

Similarly, a cost for engineering management and support is estimated as a percentage of the hardware costs; i.e.,

$$
C_{\text {engin }}=0.125 C_{\text {hardware }} \text {. }
$$

Supplies and equipment used for engineering and installation increase the total cost by $10 \%$;

$$
\text { Colee }=0.1 C_{\text {liardware }} \text {. }
$$

Adding these installation costs to the lardware cost yields a total cost which includes $\sim 10 \%$ contingency distributed (unevenly) anong the various cost centers. 


\section{REFERENCES}

[1.] P. B. Wilson, "High Energy Electron Lina:s," SLAC-PUB-4295, (195?); also contained in the Proccedings of the 1981 High Energy Particle Accelerator Summer School published by the American Institute of Physics.

[2.] P.B. Wilson, "Linear Accelerators for TeV Colliders", SLAC-PUB-3674 (Rev.) (1985)

[3.] R. Palmer, "The Interdependence of Parameter for TeV Linear Colliders", SLAC-PUB-4295 (1987)

[4.] U. Amaldi, "Introduction to the Next Generation of Linear Colliders", CERN Report EP/87-28 August 1987.

[5.] R. Bonifacio, C. Pellegrini, and N. Narducci, Free Electron Generation of Extreme Ultraviolet Coherent Radiation, ed. J. Madey and C. Pellegrini (Am. Inst. Phys., New York, 1984) p. 236.

[6.] W. A. Barletta and A.M. Sessler, Radiation from Finc, Intense Self-Focussed Beams at High Energy, UCRL-98767, in preparation

[7.] Proceedings of the ICFA Workshop on Low Emittance $e^{-} e^{+}$Beams, Brookhaven National Laboratory, March 1987, BNL-52090 (1987).

[8.] R. Sheffield, et al., Ibid. p.141

[9.] J. S. Fraser, et al., Proceedings of the 1987 IEEE Particle Accelerator Conference, p. 1705.

[10.] R. Steining, The Status of the Stanford Linenr Collider, Procendings of the 1987 Particle Accelerator Conference, p. 1.

[11.] H. Winick, Nuc. Instr. and Meth., A261, 9 (1987). 
[12.] J.W. Wang, V. Nguyen-Tuong, and G. A. Loew, "RF Breakdown Studies in a SLAC Disk-loaded Structure", 1986 Linear Accelerator Conference Proceedings, SLAC-RPT-303, 461 (1986)

[13.] 2. D. Farkas, P. B. Wilson, "Comparison of High Group Velocity Structures", SLAC-PUB-4088 (1987)

[14.] 2. D. Farkas, "The Roles of Group Velocity, Frequency and Aperture in Traveling Wave Linear Accelerator Design", SLAC internal report AAS Note 33 (1987)

[15.] R. Ruth, "Emittance Preservation", SLAC-PUB-4436 (1987); The technique analyzed by Ruth was first described by V. E. Balakin, A. V. Noppvokhatskii, V. P. Smirnov, Transverse Beam Dynamics, Proceedings of 6th National Conference on Accelerators, Dubna, 1978.

[16.] K. Halbach and R.Holsinger, Particle Accel. 7, 213 (1976)

[17.] K. Yokoya, SLAC internal report AAS-Note 26 (February, 1987).

[18.] A.C. Paul, private communication (1987).

[19.] see, for example, Proceedings of Workshop on New Developments in Particle, ed. S. Turner, CERN 87-11 (1987).

[20.] A.M. Sessler and S.S. Yu, "Relativistic Kilystron Two-Beam Accelcrator", Plyys. Rev. Lett. 58, 2439 (1986).

[21.] M. Allen et al., SLAC-PUB-4050 and UCRL-98843 (June 1988) submitted to Procecdings of European Particle Accelerator Conference (1988).

[22] W. A. Barletta, "Cost Optimization of Induction Linac Drivers of Lincar Colliders", LLNL Report UCRL-95909 (1980). Proceedings of the 3rd International Symposium on Ultra High Energy Accelerators, Orsay (1087). 
[23] D. L. Birx, L.L. Reginato, J. A. Schmidt, "Investigation into the Repetition Rate Limitations of Magnetic Switches", LLNL report UCRL-87278 (1982). 